\title{
Fred M. Donner, Muhammad and the Believers: At the Origins of Islam
}

Cambridge, Massachussets, The Belknap Press of Harvard University

Press, 2010, 280 p.

\section{Hassan Bouali}

\section{(2) OpenEdition}

Journals

Édition électronique

URL : http://journals.openedition.org/assr/25460

DOI : $10.4000 /$ assr. 25460

ISSN : $1777-5825$

Éditeur

Éditions de l'EHESS

Édition imprimée

Date de publication : 30 décembre 2013

Pagination : 182

ISSN : 0335-5985

\section{Référence électronique}

Hassan Bouali, «Fred M. Donner, Muhammad and the Believers: At the Origins of Islam », Archives de sciences sociales des religions [En ligne], 164 | 2013, mis en ligne le 21 février 2014, consulté le 21 septembre 2020. URL : http://journals.openedition.org/assr/25460 ; DOI : https://doi.org/10.4000/ assr. 25460

Ce document a été généré automatiquement le 21 septembre 2020.

(c) Archives de sciences sociales des religions 


\section{Fred M. Donner, Muhammad and the Believers: At the Origins of Islam}

Cambridge, Massachussets, The Belknap Press of Harvard University Press, 2010, $280 \mathrm{p}$.

Hassan Bouali

\section{RÉFÉRENCE}

Fred M. Donner, Muhammad and the Believers: At the Origins of Islam, Cambridge, Massachussets, The Belknap Press of Harvard University Press, 2010, 280 p. 
1 Le livre de Fred Donner est un événement historiographique important dans le champ des études portant sur l'histoire des débuts de l'Islam. Comme le titre le laisse à penser, il ne s'agit pas d'un énième livre sur le Prophète Muhammad (citons à ce titre la biographie classique de Montgoméry Watt en deux volumes, Muhammad at Mecca, 1953 ; Muhammad at Medina, 1956). L'historien américain, professeur à l'Université de Chicago, propose au contraire, dans ce livre composé de cinq parties, une histoire du mouvement religieux fondé par Muhammad.

Le premier chapitre pose, comme il est traditionnellement de mise, le cadre politique, religieux et culturel dans lequel l'Islam est apparu. L'Orient est alors

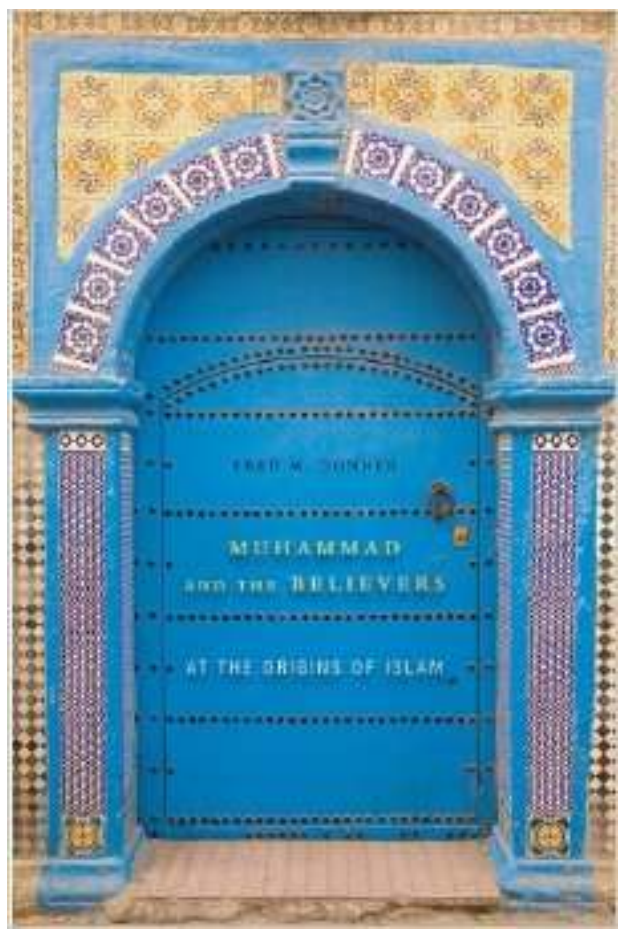
dominé par les deux «superpuissances » de l'époque, l'Empire perse sassanide et l'Empire byzantin. C'est dans le Hijâz, plus précisément à La Mecque, dans la tribu de Quraysh, au sein de cette Arabie située « entre les deux superpuissances » que Muhammad serait né vers 570.

3 C'est autour de 610 qu'il aurait reçu la première révélation de la part de l'Archange Gabriel, d'après les sources narratives islamiques. Il doit néanmoins attendre l'année 8/630 pour entrer victorieusement dans sa cité natale, La Mecque. Faut-il pour autant prendre pour argent comptant les informations données par les sources musulmanes? Fred Donner rappelle ainsi les problèmes spécifiques de ses sources, lesquelles sont toutes tardives et postérieures aux faits évoqués. Cela ne facilite donc en rien l'accès au Muhammad historique (Sur cette question, on peut se reporter à l'article de F. E. Peters, "The Quest of the Historical Muhammad", International Journal of Middle East Studies, vol. 23, $\mathrm{n}^{\circ} 3,1991$, p.91-315). Beaucoup de questions cruciales restent de fait sans réponses pour l'historien moderne : « Pourquoi les païens de Médine se rallièrent-ils si rapidement au message de Muhammad alors que les Mecquois le rejetèrent vigoureusement? Quel était le statut de Muhammad à Médine? Quelles relations entretint-il avec les Juifs de Médine?»(p.52). En dépit de ce constat, Fred Donner n'en demeure pas moins convaincu que la trame des événements se base sur des faits historiques.

Pour dépasser le cadre idéalisé esquissé par les akhbârs (récits/traditions), une source de choix s'avère indispensable pour retracer les caractéristiques du mouvement religieux fondé par Muhammad, le Coran. Suivant cette lecture, Donner réfute l'utilisation anachronique du terme de musulman, qui apparaît environ soixante-dix fois dans le Coran. Il faut lui préférer celui de Croyant, présent plus de mille fois dans le texte sacré de l'Islam. C'est à la faveur des traditionnistes que le premier terme se serait imposé un siècle après la mort du Prophète. Là encore, c'est le Coran qui sert de point d'appui à la démonstration étymologique de Donner, tout en déterminant les bases de la croyance du mouvement de Muhammad : «Vertueux sont ceux qui croient en Dieu et 
au jour dernier, aux Anges, au Livre et aux prophètes, qui donnent pour l'amour de Dieu des secours à leurs proches, aux orphelins, aux nécessiteux, aux voyageurs indigents et à ceux qui demandent l'aide, et pour délier les jougs, qui observent la prière, qui font l'aumône.» (Coran $2: 177$, p. 63)

L'hétérogénéité confessionnelle est par ailleurs un autre trait saillant du mouvement des Croyants. À suivre Donner, rien ne permet d'affirmer qu'il se soit perçu comme un groupe religieux distinct des autres communautés monothéistes du Proche Orient.

6 Le troisième chapitre revient sur l'expansion du mouvement des Croyants. Les conquêtes de La Mecque puis de Taïf, sous l'impulsion de Muhammad qui a su agréger des forces suffisantes autour de lui, signent le véritable point de départ de cette expansion territoriale (voir l'ouvrage pionnier de Fred Donner, The Early Islamic Conquests, 1981). Sa mort en 632 laisse néanmoins un vide considérable, poussant certaines tribus à faire sécession. C'est dans ce contexte tumultueux qu'Abu Bakr est nommé Commandeur des Croyants, l'étymologie du mot "amir» (commandant) révélant d'ailleurs, à bien des égards, le caractère militaire de la charge. La Umma sort finalement renforcée de ces guerres de la Ridda (mouvement d'apostasie qui s'est développé en Arabie après la mort du Prophète), notamment grâce au rôle déterminant de Khalid b.Walid, un converti tardif. Médine devient, dans ce contexte, la capitale d'un proto-empire, sa souveraineté s'étendant à toute la péninsule arabique, au point d'atteindre les frontières sassanides et byzantines. Ces dernières servirent par la suite de points d'appui aux conquêtes des règnes de Umar puis de Uthmân. Fred Donner bat d'ailleurs en brèche la thèse de la " conquête violente » du Croissant fertile. Plusieurs arguments jouent contre ce modèle qui a tant prévalu dans l'historiographie. C'est particulièrement le cas de l'archéologie, qui témoigne du faible nombre de destructions dans les cités du Proche-Orient. L'œcuménisme du nouveau mouvement religieux lui permit, en vérité, d'être bien accueilli, en des lieux où le monothéisme était solidement implanté. Dans ce cadre, les conquérants arabes se présentèrent certainement comme les promoteurs d'un monothéisme réformé, et non pas comme les tenants d'une véritable révolution religieuse. Ce qui expliquerait l'accueil favorable qui fut généralement octroyé au mouvement des Croyants. Des questions demeurent néanmoins sans réponses certaines. Comment la prétention du statut prophétique de Muhammad fut-elle reçue et comprise par les communautés juives, chrétiennes et samaritaines du Proche-Orient ? Les sources chrétiennes contemporaines à la conquête islamique apportent un semblant de réponse. Elles qualifient Muhammad de leader ou de roi par exemple. Ce n'est que dans la seconde moitié du viII siècle, que ces mêmes sources précisent que les partisans de Muhammad le désignent comme un Prophète. La documentation relative à la shahada (profession de foi) donne également un éclaircissement notable. La seconde partie de cette profession de foi, qui affirme que "Muhammad est le Messager de Dieu », est complètement absente des sources les plus anciennes. Seule la première partie, celle qui atteste qu' « il n'y a de Dieu que Dieu » (p.112) est présente dans ces sources. L'auteur y voit le reflet du pragmatisme des Croyants arabes, qui n'ont gardé que la première partie, afin de rendre leur message plus acceptable aux communautés juives et chrétiennes du Proche-Orient.

7 L'enquête se focalise ensuite sur la période 655-692 (34/655-73/692), marquée par une lutte acharnée pour le pouvoir, dans le cadre des deux premières fitnas (discordes). Sans écarter les facteurs socio-économiques qui ont pu mener à ces troubles, Donner reste persuadé que la piété en est l'élément le plus déterminant. C'est à l'issue des 
accusations d'innovation et d'impiété que le Commandeur des Croyants Uthmân est assassiné en pleine cité de Médine, sans que les habitants de la "cité du Prophète " interviennent pour empêcher cet acte. C'est aussi au nom de la piété que la lutte pour le pouvoir sera orchestrée par la suite. Les Kharijites, qui représentent selon l'auteur le mouvement le plus représentatif de ce que pouvait être l'Islam premier, considéraient par exemple que le pouvoir ne pouvait revenir qu'au plus pieux des Croyants. Le principe de la précellence en Islam pour prétendre au pouvoir suprême est tout aussi révélateur, tout comme celui de la légitimité de la famille du Prophète. L'échec de ces vues, à la suite de la victoire de Mu'awiya, explique la recrudescence de la lutte, à la mort de celui-ci. Ce n'est qu'en 692 (73/692), que les Omeyyades parviennent à vaincre l'anticalife Ibn al-Zubayr, non sans avoir manqué auparavant d'éliminer al-Husayn, le petit-fils du Prophète.

Le livre se clôt sur un chapitre qui analyse l'évolution religieuse du mouvement des Croyants. Le calife omeyyade Abd al-Malik (voir le petit ouvrage biographique de Chase F. Robinson, 'Abd al-Malik, 2007) semble avoir joué un rôle fondamental en la matière. Il reprit à son compte l'agenda des Croyants, qui fut interrompu, au gré des deux guerres civiles. Les conquêtes orchestrées sous son égide sont révélatrices de cet état d'esprit. Profondément marqué par l'eschatologie et l'arrivée imminente du Jugement dernier, Abd al-Malik entendit, par cette politique d'expansion, établir l'ordre de Dieu sur terre et devenir de ce fait le dernier souverain vertueux. Khalifat Allah (lieutenant de Dieu) devint, à cette occasion, la titulature privilégiée pour désigner le Commandeur des Croyants. Ce concept coranique s'impose ainsi au détriment de celle de Amir al Mu'minin pour affirmer la légitimité de la dynastie régnante. C'est aussi à ce moment-là que l'œcuménisme, qui avait tant caractérisé le groupe religieux jusqu'alors, prend une nouvelle orientation grâce à une redéfinition des mots-clés. Les termes de «musulman» et de "croyant» qui étaient jusque-là marqués par une subtile différence, deviennent alors synonymes. Tout porte à croire qu'il s'agit d'une réaction à la non-reconnaissance du statut prophétique de Muhammad, par les communautés monothéistes du Proche-Orient. On observe en tout cas, une amplification de la figure prophétique de Muhammad dès le dernier quart du viI ${ }^{\mathrm{e}}$ siècle ; c'est particulièrement vrai dans la documentation officielle, mais aussi, dans les hadiths (traditions prophétiques) en voie de circulation. La construction du Dôme du Rocher semble aussi s'inscrire dans cette affirmation d'une identité strictement musulmane qui marque sa différence, à titre d'exemple, avec le christianisme nestorien: "Les inscriptions du dôme du rocher incluent une sélection particulièrement marquée de versets, pour affirmer le rejet total de la doctrine trinitaire, et renforcer ainsi l'inviolabilité de l'unicité divine. [...] Il semble clair qu'Abd al-Malik et ses conseillers souhaitèrent délivrer un message puissant [...] en reconsidérant le statut des Chrétiens dans la Communauté des Croyants. » (p. 213)

9 La question des pratiques cultuelles interroge aussi l'auteur. S'il ne fait aucun doute que les rites du mouvement des Croyants remontent bien à l'époque de Muhammad, il n'en reste pas moins que des pratiques, telles que la prière ou le pélèrinage semblent avoir connu certaines évolutions.

10 Le livre de Fred Donner ouvre d'importantes perspectives historiographiques pour comprendre l'islam des origines. L'historien y développe les idées qu'il a de longue date promues dans ses travaux (voir Donner, Narratives of Islamic Origins, 1998 ; voir aussi son article « From Believers to Muslims: Confessional Self-Identity in The Early Islamic-Community 
", Al-Abhath 50-51, 2002-2003, p. 9-53). Ce livre poursuit également la réfutation des thèses du courant révisionniste, dont le chef de file, John Wansbrough (suivi par ses élèves P. Crone et M. Cook, auteurs du fameux et non moins controversé Hagarism The Making of The Islamic World, 1977), a promu les idées, en considérant l'histoire des origines de l'islam comme l'histoire mythique et tardive d'une secte juive qui a utilisé les outils de l'Ancien Testament pour s'approprier un passé inventé. Wansbrough critiqua ainsi, de manière radicale les "convictions courantes" (cité par Françoise Micheau, Les débuts de l'slam. Jalons pour une nouvelle histoire, p. 105) qui circulaient sur l'histoire du Coran, en considérant le texte sacré des Musulmans comme un ensemble de logias d'origines diverses (Wansbrough, Quranic Studies, 1977) fixé au plus tard à la fin du $\mathrm{II}^{\mathrm{e}}$ siècle de l'Hégire. Le livre de Donner s'attache donc à prouver le contraire. Non, le Prophète Muhammad n'est pas qu'une simple invention fictive. Non le texte coranique n'a pas été fixé à une date aussi tardive que celle proposée par Wansbrough ; il a au contraire été scellé très tôt, au VII siècle de l'ère chrétienne. Oui, une histoire des débuts de l'Islam est possible. Comment y aboutir? L'usage du Coran en tant que source, est un élément crucial de la méthodologie de Donner. En prenant le parti d'affirmer que le Coran a été fixé très tôt, dès le vile siècle, Fred Donner affirme donc qu'il est possible d'utiliser le texte sacré de l'Islam, pour tenter de comprendre le mouvement religieux des Croyants. Les sources chrétiennes, mais aussi l'archéologie, permettent aussi de retracer les contours historiques du mouvement de Muhammad. À ce titre, le livre de Fred Donner s'inscrit pleinement dans une démarche propre à l'anthropologie religieuse, ce qui est un fait notable, du fait même de l'indigence de cette dimension, dans l'historiographie des débuts de l'Islam. Un autre point fort du livre de Donner est à noter. Il est illustré par quelques photographies intéressantes (des pièces de monnaie, une photographie de la Kaaba, celles de vestiges chrétiens), et des versets coraniques intégrés au texte pour étayer l'argumentation. Deux appendices qui portent respectivement sur une traduction de la Charte de Yathrib et sur les inscriptions coraniques du Dôme du Rocher sont donnés à la fin de l'ouvrage. La bibliographie, qui suit un cheminement propre aux différents chapitres du livre, est commentée. C'est somme toute un ouvrage destiné à tout type de public et qui mériterait une traduction en français. 